\title{
El papel de la medicina forense en el contexto del trabajo humanitario
}

\section{Rol of forensic medicine in the context of humanitarian work}

\section{Resumen}

Actualmente la medicina forense tiene campos de acción más amplios e incluye el campo humanitario. Las Convenciones de Ginebra y los protocolos adicionales incluyen reglas claras referentes al manejo de los cadáveres y la responsabilidad de las diferentes autoridades o grupos en conflicto para identificarlos y disponer de ellos, de manera que sea posible recuperarlos más tarde y entregarlos a sus familias. El Comité Internacional de la Cruz Roja ha sido la organización pionera en estas labores, e igualmente Naciones Unidas e INTERPOL, entre otras organizaciones públicas, privadas y ONGs; emplean actualmente protocolos y guías de trabajo para contextos de atención humanitaria y prevención de violaciones al Derecho Internacional Humanitario. El empleo de la medicina forense en diferentes partes del mundo ha sido y seguirá siendo un componente importante de las misiones humanitarias.

Palabras clave: Medicina Forense. Trabajo Humanitario. Convenciones de Ginebra. Ley Internacional Humanitaria.

\section{Abstract}

Today the forensic medicine has broader action fields including the humanitarian activities. The Geneva Conventions and additional protocols have clear rules about the management of dead bodies and the responsibility of many authorities or armed groups in conflict in order to identify those bodies and to deal with them in such manner that facilitate late recovery and to bring them back to their relatives. The International Committee of the Red Cross has been the pioneer organization in those tasks, also United Nations and INTERPOL, among other public and private organizations and NGOs, are applying protocols and guidelines to jobs in the humanitarian context and prevention of violations of the International Humanitarian Law. The use of forensic medicine around the world has been and will continue being an important component of humanitarian missions.

Key words: Forensic Medicine. Humanitarian Work. Geneva Conventions. International Humanitarian Law.

Tradicionalmente las definiciones de medicina forense $o$ de ciencias forenses implican que tienen como campo de acción principal la investigación de casos criminales. Una frecuente descripción de patología forense indica que es una rama de la medicina que aplica los principios y conocimientos de las ciencias médicas a problemas en el campo de la Ley ${ }^{1}$, pero cada vez se observan más aplicaciones de las especialidades forenses a otros campos diferentes a la investigación criminal o al apoyo de procesos judiciales.

Recientemente se han aplicado conocimientos forenses a investigaciones arqueológicas o a análisis de hechos de interés histórico, sin pretender obligatoriamente llevar los dictámenes ante tribunales judiciales; e igual desde hace aproximadamente 10 años se ha impulsado con especial fuerza el uso de las ciencias forenses como apoyo al trabajo humanitario, específicamente con el objetivo de hacer un adecuado manejo de las víctimas de conflictos y de desastres.

Los contextos geográfico, histórico, político y cultural del trabajo humanitario tienen, entre otras, las siguientes características comunes: el objetivo principal es el respeto de los derechos fundamentales de los seres humanos, independientemente de su raza,
MA. Duque

Médico Cirujano, especialista en Medicina Forense y Antropología Forense. Director General de MD FORENSIC CONSULTANTS. Bogotá D.C., Colombia.

Exdirector General del Instituto Nacional de Medicina legal y Ciencias Forenses de Colombia. Asesor forense y perito en misiones de la Organización de las Naciones Unidas y del Comité Internacional de la Cruz Roja.

Correspondencia: Dr. Máximo A.Duque Carrera 7 No 156-78 North Pont Bussines Center

Oficina 806 Bogotá D.C. Colombia E-mail: maximoduque@ mdforensic.com

Fecha de recepción: 24.MAR.2010

Fecha de aceptación: 24.MAR.2010 
sexo, creencia religiosa, nacionalidad, filiación política o edad. La prioridad es aliviar el sufrimiento de los individuos siguiendo como único criterio las necesidades básicas que ellos tienen. La protección de las víctimas es fundamental para evitar que se agrave su condición o que se incremente su número.

Las organizaciones que tienen como propósito el trabajo humanitario siguen lineamientos generales que han sido principalmente establecidos por el Comité Internacional de la Cruz Roja (CICR) y que paulatinamente han sido extrapolados o adaptados al trabajo de cada entidad. El Comité Internacional de la Cruz Roja es una organización suiza privada, imparcial, neutral e independiente que se fundó en 1863 y tiene como misión fundamental proteger y asistir a las víctimas de conflictos armados actuando estrictamente como una organización neutral e imparcial ${ }^{2}$.

Las tareas del CICR incluyen visitar prisioneros de guerra y civiles detenidos; búsqueda de personas desaparecidas; transmisión de mensajes entre miembros de familias separadas por el conflicto; reunificación de familias dispersas; provisión de alimento, agua y asistencia médica a civiles sin acceso a estos servicios básicos; divulgación de conocimientos sobre derecho humanitario; monitoreo del cumplimiento y desarrollo del derecho humanitario; búsqueda de atención a casos de violaciones del derecho humanitario² .

El grupo de reglas que fueron establecidas posteriormente a la fundación del CICR, con el objetivo de proteger a las víctimas de los conflictos armados, y que han sido ratificadas prácticamente por todas las naciones del mundo, son conocidas como el Derecho Internacional Humanitario y los denominados Convenios de Ginebra del 12 de agosto de 1949 son, en la actualidad, su base más importante, sin desconocer otros tratados con similares objetivos.

\section{Convenios de Ginebra del 12 de agosto de 1949: aspectos relevantes a las prác- ticas forenses y manejo de fallecidos}

El I Convenio de Ginebra está dedicado a aliviar la suerte que corren los heridos y los enfermos de las fuerzas armadas en campaña ${ }^{3}$. Hay varios artículos hablando específicamente de los muertos en el conflicto que vale la pena citar: En su artículo 15 reza: "En todo tiempo, y especialmente después de un combate, las partes en conflicto tomarán sin tardanza todas las medidas posibles para buscar y recoger a los heridos y a los enfermos, para protegerlos contra el pillaje y los malos tratos y proporcionarles la asistencia necesaria, así como para buscar a los muertos e impedir que sean despojados (...)". En el artículo 16 establece que "Las partes en conflicto deberán registrar, tan pronto como sea posible, toda la información adecuada para identificar a los heridos, a los enfermos y a los muertos de la parte adversaria caídos en su poder (...)Las Partes en conflicto redactarán y se comunicarán, por el conducto indicado en el párrafo anterior, las actas de defunción o las listas de fallecimientos debidamente autenticadas. Recogerán y se transmitirán también, por mediación de la misma oficina, la mitad de la doble placa de identidad, los testamentos u otros documentos que tengan importancia para la familia de los fallecidos, el dinero y, en general, cuantos objetos de valor intrínseco o afectivo se hayan encontrado sobre los muertos. Estos objetos, así como los no identificados, serán remitidos en paquetes lacrados, acompañados de una declaración con todos los detalles necesarios para la identificación del poseedor fallecido, así como de un inventario completo del paquete."

Los artículos anteriores claramente indican que los fallecidos y sus familias son víctimas, no enemigos, y por tanto hay derechos que deben ser respetados, y específicamente el artículo 17 puntualiza lo siguiente: "Prescripciones relativas a los muertos. Servicio de tumbas. Las Partes en conflicto velarán por que la inhumación o la incineración de los cadáveres, hecha individualmente en la medida en que las circunstancias lo permitan, vaya precedida de un atento examen y, si es posible, médico de los cuerpos, a fin de comprobar la muerte, determinar la identidad y poder dar cuenta al respecto. La mitad de la doble placa de identidad o la placa misma, si se trata de una placa sencilla, quedará sobre el cadáver. Los cuerpos no podrán ser incinerados más que por imperiosas razones de higiene 0 por motivos basados en la religión de los fallecidos. En caso de incineración, se hará la correspondiente mención detallada indicando los motivos en el acta de defunción o en la lista autenticada de fallecimientos. Además, las Partes en conflicto velarán por que se entierre a los muertos honrosamente, si es posible según los ritos de la religión a la que pertenecían, porque sus sepulturas sean respetadas, agrupadas, si es posible, de conformidad con la nacionalidad de los fallecidos, convenientemente atendidas y marcadas de modo que siempre puedan ser encontradas. Para ello, organizarán, al comienzo de las hostilidades, un Servicio oficial de tumbas, a fin de permitir exhumaciones eventuales, garantizar la identificación de los cadáveres, sea cual fuere el lugar de las sepulturas, y su eventual traslado al respectivo país de origen. Estas disposiciones son 
igualmente aplicables a las cenizas, que serán conservadas por el Servicio de tumbas, hasta que el país de origen comunique las medidas que desea tomar a este respecto. En cuanto las circunstancias lo permitan y, a más tardar, al fin de las hostilidades, estos servicios se intercambiarán, por mediación de la oficina de información mencionada en el párrafo segundo del artículo 16, listas en las que se indiquen exactamente el lugar y la designación de las tumbas, así como los datos relativos a los muertos en ellas sepultados"

El II Convenio de Ginebra se aplica a aliviar la suerte que corren los heridos, los enfermos y los náufragos de las fuerzas armadas en el mar ${ }^{4}$. El artículo 5 se refiere a "Aplicación por las Potencias neutrales. Las Potencias neutrales aplicarán, por analogía, las disposiciones del presente Convenio a los heridos, a los enfermos y a los náufragos, a los miembros del personal sanitario y religioso pertenecientes a las fuerzas armadas de las Partes en conflicto que sean recibidos o internados en su territorio, así como a los muertos recogidos". Luego, los artículos 19 y 20 hablan puntualmente del registro de los heridos, náufragos, enfermos y muertos, así como de la disposición de los cadáveres, y vale la pena enfatizar el texto del artículo 20: "Prescripciones relativas a los muertos. Las Partes en conflicto se cerciorarán de que a la inmersión de los muertos, efectuada individualmente en la medida en que las circunstancias lo permitan, preceda un minucioso examen, médico si es posible, de los cuerpos, a fin de comprobar la muerte, determinar la identidad y poder informar al respecto. Si se utiliza la doble placa de identidad, la mitad de la misma quedará sobre el cadáver. Si se desembarca a los muertos, les serán aplicables las disposiciones del Convenio de Ginebra del 12 de agosto de 1949 para aliviar la suerte que corren los heridos y los enfermos de las fuerzas armadas en campaña".

El III Convenio de Ginebra es relativo al trato debido a los prisioneros de guerra ${ }^{5}$, tiene como antecedente un Convenio firmado en 1929, y vale la pena citar en su integridad los artículos 120 y 121 que rezan: "Sección III - Fallecimiento de prisioneros de guerra. Artículo 120 - Testamentos, actas de defunción, inhumación, incineración. Los testamentos de los prisioneros de guerra se redactarán de modo que reúnan las condiciones de validez requeridas por la legislación de su país de origen, el cual tomará las medidas necesarias para poner dichas condiciones en conocimiento de la Potencia detenedora. Tras solicitud del prisionero de guerra, y en todo caso después de su muerte, el testamento será transmitido sin demora a la Potencia protectora; una copia, certificada como fiel, será remitida a la Agencia
Central de Información. Los certificados de defunción de conformidad con el modelo anejo al presente Convenio, o listas, firmadas por un oficial encargado de todos los prisioneros de guerra muertos en cautiverio, serán remitidos, en el más breve plazo, a la Oficina de Información de los Prisioneros de Guerra instituida según el artículo 122. Los datos de identificación cuya lista figura en el tercer párrafo del artículo 17, el lugar y la fecha del fallecimiento, la causa de éste, el lugar y la fecha de inhumación así como toda la información necesaria para identificar las tumbas, deberán figurar en esos certificados o en esas listas. Al entierro o a la incineración deberá preceder un examen médico del cadáver para comprobar el fallecimiento, posibilitar la redacción de un informe y, si procede, identificar al difunto. Las autoridades detenedoras velarán por que los prisioneros de guerra fallecidos en cautiverio sean enterrados honrosamente si es posible según los ritos de la religión a la que pertenecían, y por que las tumbas sean respetadas, decentemente mantenidas y marcadas de modo que siempre puedan ser reconocidas. Siempre que sea posible, los prisioneros de guerra fallecidos que dependían de la misma Potencia serán enterrados en el mismo lugar. Los prisioneros de guerra fallecidos serán enterrados individualmente, excepto en caso de fuerza mayor que imponga una tumba colectiva. Los cadáveres no podrán ser incinerados más que si imperiosas razones de higiene o la religión del faIlecido lo requieren, o si éste expresó tal deseo. En caso de incineración, se hará constar en el acta de defunción, con indicación de los motivos. A fin de que siempre puedan encontrarse las tumbas, habrá de registrar todos los datos relativos a éstas y a las inhumaciones el Servicio de Tumbas instituido por la Potencia detenedora. Serán transmitidos a la Potencia de la que dependían estos prisioneros de guerra las listas de las tumbas y los datos relativos a los prisioneros de guerra enterrados en cementerios o en otro lugar. Incumbirá a la Potencia que controle el territorio, si es Parte en el Convenio, cuidar dichas tumbas y registrar todo traslado ulterior de los cadáveres. Estas disposiciones se aplican también a las cenizas, que serán conservadas por el Servicio de Tumbas hasta que el país de origen comunique las disposiciones definitivas que desea tomar a este respecto.

Artículo 121 - Prisioneros muertos o heridos en circunstancias especiales. Toda muerte o toda herida grave de un prisionero de guerra, causada, o que haya sospecha de haber sido causada, por un centinela, por otro prisionero de guerra o por cualquier otra persona, así como todo fallecimiento cuya causa se ignore será inmediatamente objeto de un investigación oficial por parte de la Potencia 
detenedora. Acerca de este asunto se informará inmediatamente a la Potencia protectora. Se recogerán las declaraciones de los testigos, especialmente las de los prisioneros de guerra; se remitirá a dicha Potencia un informe en el que éstas figuren. Si la investigación prueba la culpabilidad de una o de varias personas, la Potencia detenedora tomará las oportunas medidas para incoar diligencias judiciales contra el responsable o los responsables."

A lo anterior se suman desarrollos más recientes consignados en el artículo 32 del Protocolo Adicional I de 1977 que hace referencia al derecho de las familias a conocer la suerte que han corrido sus familiares $^{2,6}$. Esto va en concordancia con el IV Convenio de Ginebra, específicamente relativo a la protección de los civiles en tiempo de guerra.

\section{Desarrollo posterior del uso de las ciencias forenses en el marco de las actividades humanitarias}

Con los anteriores artículos se establece que la identificación de los cuerpos, tanto de civiles como de miembros de las fuerzas armadas en conflicto, su manejo digno y su correcta disposición temporal o permanente son tareas humanitarias. Para cumplirlas, el desarrollo científico forense ofrece hoy en día diversas herramientas como la genética forense, antropología forense, sistemas de identificación por carta dental, dactiloscopia forense, entre otras.

Igualmente el manejo digno de los civiles detenidos y prisioneros de guerra conlleva actividades de diagnóstico y prevención de fenómenos como la tortura, los tratos no dignos o degradantes y el respeto a los civiles especialmente vulnerables, como mujeres, menores de edad, ancianos y personas discapacitadas.

Los Convenios de Ginebra fueron redactados poco después de finalizada la II Guerra Mundial, cuando tecnologías como la genética forense y otros sistemas de identificación de cuerpos no se habían desarrollado al nivel que hoy conocemos. En la actualidad es posible identificar cuerpos humanos de fallecidos muchos años atrás, de manera que está proscrito incinerar cuerpos o lanzarlos al mar o sepultarlos indiscriminadamente, dado que es posible conservarlos para finalmente entregarlos debidamente identificados e individualizados a sus familiares. Un cuerpo sin identificar significa un desaparecido para alguna familia.

En 2001 el CICR, atendiendo el clamor de las comunidades por buscar una solución al problema de los desaparecidos, lanzó el proyecto The Missing (Los Desaparecidos). Posteriormente, en 2003, se sentaron las bases de este proyecto cuyo objetivo es impulsar la conciencia entre el público en general, gobernantes, militares, organizaciones nacionales e internacionales (incluyendo todo el movimiento de las sociedades nacionales de la Cruz Roja y de la Media Luna Roja) sobre la tragedia de las personas desaparecidas como resultado de conflictos armados o violencia interna y sobre la angustia de sus familias. Para ello se buscaría crear y poner a disposición herramientas para la acción y comunicaciones de datos, de manera que se gestione de parte de las autoridades la responsabilidad de resolver el problema de los desaparecidos, asistir de la mejor manera posible sus familias y prevenir futuras desapariciones.

Con ese importante mandato el CICR ha creado un equipo forense capaz de proveer cursos de formación en ciencias forenses, excepcionalmente realizar labores forenses directamente, asesorar la práctica forense en cualquier parte del mundo y generar herramientas tecnológicas de fácil uso en contextos de conflicto o desastre. Igualmente este mandato ha servido de base para organizaciones de carácter privado o público, así como organizaciones no gubernamentales, que propenden por un adecuado manejo de los cadáveres y de la información que de las familias de desaparecidos se puede obtener para la búsqueda de sus seres queridos. A la par muchas organizaciones han llevado la práctica forense al campo de la atención de desastres de origen natural y se han generado protocolos interesantes como los que actualmente usa la Organización Internacional de Policía Criminal -INTERPOL7?.

Simultáneamente se han desarrollado protocolos internacionales para el manejo apropiado de los cadáveres y para conducir las actividades forenses con mínimos estándares de calidad, que permitan obtener resultados positivos en la identificación de cuerpos y búsqueda de desaparecidos, así como en el estudio de casos de posible tortura.

Entre estos protocolos, cabe destacar el denominado Protocolo de Estambul: Manual para la investigación y documentación eficaces de la tortura y otros tratos o penas crueles, inhumanos o degradantes ${ }^{8}$. Este manual fue diseñado por expertos de gran experiencia en la materia y busca establecer un método científico para la investigación de casos de posible tortura. Por otra parte, el Protocolo de Minnesota: manual de las Naciones Unidas para la prevención e investigación eficaces de las ejecuciones ilegales arbitrarias y sumarias ${ }^{9}$, es igualmente un documento de gran importancia para los investigadores forenses a cargo de casos que puedan caer en el ámbito de 
las violaciones a los derechos humanos, por ejemplo en casos de personas que fallecen bajo custodia de la autoridad ${ }^{10}$.

En los cinco continentes se han llevado a cabo misiones forenses humanitarias, por parte de varias organizaciones, para identificar cadáveres, buscar desaparecidos y atender desastres. Algunas de las experiencias, entre muchas otras, que pueden mencionarse son la asesoría forense a Iraq durante los últimos 5 años, tiempo en el cual el Instituto de Medicina Legal de Bagdad ha alcanzado un nivel tecnológico que le permite hacer identificaciones de cuerpos usando genética forense; exhumación de cuerpos en el sur de Arabia Saudita correspondientes a soldados muertos en las denominadas Guerras del Golfo ocurridas en 1980 y 1991 , estos cuerpos fueron repatriados y entregados a sus familias; asesoría forense a Irán con el objetivo de posibilitar la identificación de restos humanos de personas fallecidas en diferentes conflictos armados; capacitación en medicina forense a países de Medio Oriente y Norte de África con el objetivo de conseguir estándares mínimos de calidad en el manejo de cuerpos e identificación de los mismos con miras a prevenir identificaciones erróneas y disposición inadecuada de cuerpos sin información que permita identificarlos posteriormente.

En la región de los Balcanes, y específicamente en los países de la antigua Yugoslavia, se llevaron a cabo procesos de exhumación e identificación de cuerpos con la participación de equipos y expertos forenses provenientes de diversos países, por primera vez llegando inclusive al uso masivo de la genética para cotejar perfiles de ADN de familias y de restos humanos.

En Suramérica se han llevado a cabo procesos de capacitación en varios países y se ha conformado la primera red de cooperación forense internacional en la región, denominada Red Iberoamericana de Instituciones Forenses, la cual en principio permite el apoyo mutuo de las organizaciones participantes en casos de desastres. Adicionalmente en Chile, atendiendo la iniciativa de la Presidencia de la República, se llevó a cabo un proceso de asesoría con miras a lograr la identificación final de restos humanos producto de conflictos en la época de la dictadura del General Pinochet.

En Chipre se adelantan procesos de exhumación de restos humanos y búsqueda de desaparecidos producto de los conflictos que llevaron a que la población de la isla se dispersara.

De la misma manera, es notorio el esfuerzo que han realizado los equipos forenses para apoyar de manera urgente la atención de desastres naturales como el tsunami en Asia y el terremoto en Haití. E igualmente catástrofes generadas por conflictos armados en Kenia, Sudán, Afganistán y Colombia.

Las experiencias y desarrollo reciente sobre las actividades forenses humanitarias se encuentran disponibles en textos como: La Gestión de Cadáveres en Situaciones de Desastre: Guía Práctica para Equipos de Respuesta ${ }^{11}$, publicado por la Organización Panamericana de la Salud, la Organización Mundial de la Salud, el CICR y la Federación Internacional de Sociedades de la Cruz Roja y Media Luna Roja, está disponible en francés, español, inglés, árabe y farsi; Manejo de Cadáveres en Situaciones de Desastre ${ }^{12}$, igualmente disponible en varios idiomas; Missing People, DNA Analysis and Identification of Human Remains: a guide to best practice in armed conflicts and other situations of armed violence ${ }^{13}$, publicado en 2005 por el CICR.

Cumpliendo con su mandato, el CICR ha desarrollado además sistemas de manejo de información que permiten centralizar los datos personales ${ }^{6}$ para aumentar las posibilidades de cruzar la información proveniente de quienes buscan a un desaparecido con los datos de instituciones forenses. De todos los organismos humanitarios y de derechos humanos, se reconoce que el CICR, cuando está presente, es la organización idónea para centralizar tales datos recogidos con fines humanitarios. Sin embargo, debido a la misión y principios del CICR, esta información no es facilitada para procesos de instrucción judicial. Actualmente se desarrolla un software capaz de manejar esos datos en diferentes idiomas y de hacer cruces de datos provenientes de múltiples fuentes. E igualmente diversas organizaciones vienen desarrollando sistemas parecidos, entre los cuales vale la pena resaltar el SIRDEC (Sistema de Información Red de Desaparecidos y Cadáveres) creado por el Instituto Nacional de Medicina Legal y Ciencias Forenses de Colombia en 2006 y que tiene como objetivo centralizar y cruzar datos de diferentes fuentes, especialmente oficiales, para buscar desaparecidos de manera urgente y con acceso desde la web ${ }^{14}$

Es claro entonces que el campo de acción de las ciencias forenses, y específicamente de la medicina forense, es cada vez más amplio. El papel de la medicina forense en el contexto del trabajo humanitario se fundamenta en normas internacionales que han sido ratificadas casi por la totalidad de naciones, y los objetivos de este tipo de actividades son tan válidos como los que se persiguen al emplear las ciencias forenses en el contexto de las investigaciones judiciales. 


\section{Bibliografía}

1. Di Maio Vincent J, Di Maio Dominick. Forensic pathology. 2nd Edition. CRC press 2001.

2. International Committee of the Red Cross. Discover the ICRC. Septiembre 2005.

3. I Convenio de Ginebra del 12 de Agosto de 1949. Disponible en: www.icrc.org

4. II Convenio de Ginebra del 12 de Agosto de 1949. Disponible en: www.icrc.org

5. III Convenio de Ginebra del 12 de Agosto de 1949. Disponible en: www.icrc.org

6. International Committee of the Red Cross. Las personas desaparecidas y sus familiares. 2004. Disponible en: http://www.cicr.org/Web/spa/sitespaO.nsf/ $\mathrm{html} /$ 5XYLEW

7. Organización Internacional de Policía Criminal - INTERPOL. Guía de identificación de víctimas de catástrofes, 1997. Disponible en: www.interpol.int

8. Oficina del Alto Comisionado de las Naciones Unidas para los Derechos Humanos. Protocolo de Estambul: manual para la investigación y documentación eficaces de la tortura y otros tratos 0 penas crueles, inhumanos o degradantes. Ginebra: Naciones Unidas 1999.
9. Naciones Unidas. Protocolo de Minnesota. Manual de las Naciones Unidas para la prevención e investigación eficaces de las ejecuciones ilegales arbitrarias y sumarias. OACDG:(1991) UN Pub. Sales No. E.91.IV.1 (doc. ST/CSDHA/12).

10. Lasso EF, Varela VL, Pachar JV. Muerte traumática de personas privadas de libertad: descripción de un caso. Cuadernos de Medicina Forense 2009;58.

11. Pan American Health Organization, World Health Organization, International Committee of the Red Cross, International Federation of Red Cross and Red Cressent Societies. La gestión de cadáveres en situaciones de desastre: guía práctica para equipos de respuesta. Washington: Organización Panamericana de la Salud 2006.

12. Organización Panamericana de la Salud, Organización Mundial de la Salud. Manejo de cadáveres en situaciones de desastre. Washington: Organización Panamericana de la Salud 2004.

13. International Committee of the Red Cross. Missing people, DNA analysis and identification of human remains: a guide to best practice in armed conflicts and other situations of armed violence 2005. Disponible en: www.icrc.org.

14. Instituto Nacional de Medicina Legal y Ciencias Forenses, Colombia, www.medicinalegal.gov.co. 